\title{
ON UNIFORM CONVERGENCE OF PIECEWISE-LINEAR SOLUTIONS TO MINIMAL SURFACE EQUATION
}

\author{
M.A. GATSUNAEV, A.A. KLYACHIN
}

\begin{abstract}
In the paper we consider piecewise-linear solutions of the minimal surface equation over a given triangulation of a polyhedral domain. It is shown that under certain conditions, the gradients of these functions are bounded as the maximal diameter of the triangles of the triangulation tends to zero. It is stressed that this property holds if the piecewise-linear function approximates the area of the graph of a smooth function with a required accuracy. An implication of the obtained properties is the uniform convergence of piecewise linear solutions to the exact solution of the minimal surface equation.
\end{abstract}

Keywords: : piecewise-linear functions, minimal surface equation, the approximation of the area functional

Mathematics Subject Classification: 35J25, 35J93, 65N30

\section{INTRODUCTION}

Some problems appearing in designing architectural structures are reduced to constructing surfaces of minimal area. This was reflected quite in detail in book [1] and work [2], where the problem of developing of awning cloth construction was studied. The detailed analysis of the provided results leads one to the problem on developing effective methods for approximative solving the minimal surface equations and mathematical justification of the found methods in the sense of stability and convergence of approximate solutions. The main difficulty in studying such issues is that the minimal surface equation is non-linear and this is way classical methods employed for linear equations are not applicable.

Our approach is based on introducing the notion of a piecewise-linear solutions to the minimal surface equation over a given triangulation of a given domain and we establish needed properties for these solutions. Namely, we show that the accuracy order of approximation for area functional with respect to the triangles diameters equals two; we establish that the partial derivatives are bounded by a constant independent of the fineness of partition under the sufficient approximation of the area functional and so forth. The proved statements allow us to establish, in particular, the uniform convergence as the diameters of triangulation triangles tend to zero.

\section{Piece-Wise linear SOlUtion to Minimal SURFACE EQUATION}

Suppose we are given a polyhedral bounded domain $\Omega \subset \mathbb{R}^{n}$. Consider a partition of this polyhedron into non-degenerate tetrahedrons $T_{1}, T_{2}, \ldots, T_{N}$. Let $M_{1}, M_{2}, \ldots, M_{m}$ be all the vertices of these tetrahedrons. We shall assume that each of points $M_{i}$ is neither internal for any side non for any edge of the tetrahedrons.

M.A. Gatsunaev, A.A. Klyachin, On uniform convergence of piecewise-linear solutions to MINIMAL SURFACE EQUATION.

(c) Gatsunaev M.A., Klyachin A.A. 2014.

The work is supported by RFBR (grant no. 13-01-97034 r_povolzhe_a ).

Submitted November 11, 2014. 
For an arbitrary set of values $u_{1}, u_{2}, \ldots, u_{m}$ we define a piece-wise linear function $u: \Omega \rightarrow \mathbb{R}$ so that $u\left(M_{i}\right)=u_{i}, i=1, \ldots, m$ and we introduce the function $u(x)=p_{1}^{k} x_{1}+\cdots+p_{n}^{k} x_{n}+b^{k}$ on each tetrahedron $T_{k}, k=1, \ldots, N$. This function is continuous in $\Omega$ and in each tetrahedrons $T_{k}$ the gradient $\nabla u \equiv p^{k}=\left(p_{1}^{k}, \ldots, p_{n}^{k}\right)$ is well-defined. Therefore, the area under the graph of function $u$ is given by the sum

$$
S(p)=S\left(p^{1}, \ldots, p^{N}\right)=\sum_{k=1}^{N} \int_{T_{k}} \sqrt{1+|\nabla u|^{2}} d x=\sum_{k=1}^{N} \sqrt{1+\left|p^{k}\right|^{2}} v\left(T_{k}\right),
$$

where $v\left(T_{k}\right)$ is the $n$-dimensional volume of tetrahedron $T_{k}$.

Since vectors $p^{1}, \ldots, p^{N}$ are uniquely defined by values $u_{1}, \ldots, u_{m}$, we can write the value of the area $S(p)$ in terms of the variables $u=\left(u_{1}, \ldots, u_{m}\right): S(u)=S\left(u_{1}, \ldots, u_{m}\right)$. Indeed, variables $p^{1}, \ldots, p^{N}$ are expressed linearly in terms of variables $u_{1}, \ldots, u_{m}$. Then there exist numbers $a_{l i}^{k}$ such that

$$
p_{l}^{k}=\sum_{i=1}^{m} a_{l i}^{k} u_{i}, k=1, \ldots, N, l=1, \ldots, n .
$$

Coefficients $a_{l i}^{k}$ are uniquely defined by the partition of domain $\Omega$ into tetrahedrons $T_{1}, \ldots, T_{N}$. Thus,

$$
S(u)=S\left(u_{1}, \ldots, u_{m}\right)=\sum_{k=1}^{N} \sqrt{1+\sum_{l=1}^{n}\left(\sum_{i=1}^{m} a_{l i}^{k} u_{i}\right)^{2}} \cdot v\left(T_{k}\right) .
$$

Suppose that in vertices $M_{1}, \ldots, M_{m}$ we are given values $\varphi_{1}, \ldots, \varphi_{m}$. We denote by $\varphi$ the associated piecewise-linear function constructed by these values. Let us state the problem on finding a piecewise-linear function $u$ providing the minimum of area $S(u)$ and satisfying the boundary condition, i.e.,

$$
S\left(u_{1}, \ldots, u_{m}\right) \rightarrow \min , \quad u\left(M_{i}\right)=\varphi_{i}, \quad \forall M_{i} \in \partial \Omega .
$$

Remark. Let $u^{*}=\left(u_{1}^{*}, \ldots, u_{m}^{*}\right)$ be a solution to problem (1). By $u^{*}$ we shall denote the associated piecewise-linear function. Suppose that $h(x)$ is an arbitrary piecewise-linear function satisfying the condition $h\left(M_{i}\right)=0$ for each point $M_{i} \in \partial \Omega$. Then the function $\sigma(t)=S\left(u^{*}+\right.$ th $)$ achieves the minimum at the point $t=0$. Hence, $\sigma^{\prime}(0)=0$ that is equivalent to the identity

$$
\sum_{k=1}^{N} \int_{T_{k}} \frac{\left\langle\nabla u^{*}, \nabla h\right\rangle}{\sqrt{1+\left|\nabla u^{*}\right|^{2}}} d x=0 .
$$

Theorem 1. Problem (1) is uniquely solvable.

Proof. We observe that function $S\left(u_{1}, \ldots, u_{m}\right)$ is convex with respect to variables $u_{1}, \ldots, u_{m}$. And since in the boundary points the values of function $u$ are fixed,

$$
\lim _{|u| \rightarrow+\infty} S(u)=+\infty
$$

where $|u|=\max _{1 \leqslant i \leqslant m}\left|u_{i}\right|$. Hence, function $S(u)$ achieves its minimum at some point $u^{*}$.

Let us prove the uniqueness. We assume the opposite, i.e., there exists one more solution $v^{*}$ to problem (1). Then piecewise-linear function $v^{*}$ satisfies condition (2) as well. Letting $h=v^{*}-u^{*}$, we arrive at the identity

$$
\sum_{k=1}^{N} \int_{T_{k}}\left(\frac{\left\langle\nabla v^{*}, \nabla\left(v^{*}-u^{*}\right)\right\rangle}{\sqrt{1+\left|\nabla v^{*}\right|^{2}}}-\frac{\left\langle\nabla u^{*}, \nabla\left(v^{*}-u^{*}\right)\right\rangle}{\sqrt{1+\left|\nabla u^{*}\right|^{2}}}\right) d x=0 .
$$


In what follows we shall make use of the inequality

$$
\left\langle\frac{\xi}{\sqrt{1+|\xi|^{2}}}-\frac{\eta}{\sqrt{1+|\eta|^{2}}}, \xi-\eta\right\rangle \geqslant \frac{|\xi-\eta|^{2}}{\sqrt{1+|\xi|^{2}}\left(\sqrt{1+|\xi|^{2}} \sqrt{1+|\eta|^{2}}+|\xi||\eta|+1\right)},
$$

satisfied for all vectors $\xi, \eta \in \mathbb{R}^{n}$. We note that similar inequalities were obtained in works [3], [4], 5] and were also employed for studying the issues on uniqueness of solution to the minimal surface equation. Inequality (4) by means of which we shall obtain the uniqueness will be also employed for estimating the gradient of piecewise-linear solution $u^{*}$. And we can not apply the inequalities from the above cited works for this estimate. This is the reason why we employ inequality (4). We prove it as follows.

First we note that

Then

$$
\sqrt{1+|\xi|^{2}} \geqslant \sqrt{1+|\eta|^{2}}+\frac{\langle\eta, \xi-\eta\rangle}{\sqrt{1+|\eta|^{2}}}
$$

$$
\begin{aligned}
\left\langle\frac{\xi}{\sqrt{1+|\xi|^{2}}}-\frac{\eta}{\sqrt{1+|\eta|^{2}}}, \xi-\eta\right\rangle & =-\frac{\langle\xi, \eta-\xi\rangle}{\sqrt{1+|\xi|^{2}}-\frac{\langle\eta, \xi-\eta\rangle}{\sqrt{1+|\eta|^{2}}}} \\
& \geqslant \sqrt{1+|\eta|^{2}}-\sqrt{1+|\xi|^{2}}-\frac{\langle\xi, \eta-\xi\rangle}{\sqrt{1+|\xi|^{2}}} \\
& =\frac{\sqrt{1+|\xi|^{2}} \sqrt{1+|\eta|^{2}}-\langle\xi, \eta\rangle-1}{\sqrt{1+|\xi|^{2}}} \\
& \geqslant \frac{|\xi-\eta|^{2}}{\sqrt{1+|\xi|^{2}}\left(\sqrt{1+|\xi|^{2}} \sqrt{1+|\eta|^{2}}+|\xi||\eta|+1\right)}
\end{aligned}
$$

Letting $\xi=\nabla u^{*}$ and $\eta=\nabla v^{*}$ in inequality (4), by (3) we obtain that $\nabla u^{*} \equiv \nabla v^{*}$. Since on boundary $\partial \Omega$ functions $u^{*}$ and $v^{*}$ coincide, we obtain the desired identity $u^{*} \equiv v^{*}$.

\section{ESTIMATE FOR MODUlus OF GRADIENT}

Let $f$ be a solution to the minimal surface equation

$$
\sum_{i=1}^{n} \frac{\partial}{\partial x_{i}}\left(\frac{f_{x_{i}}}{\sqrt{1+|\nabla f|^{2}}}\right)=0
$$

in domain $\Omega$ continuous in $\bar{\Omega}$ and $\left.f\right|_{\partial \Omega}=\left.\varphi\right|_{\partial \Omega}$, where $\varphi(x)$ is a continuous function defined on the boundary of domain $\Omega$. It is worth mentioning that the corresponding Dirichlet problem for an arbitrary domain (even with a smooth boundary) is not always solvable. For planar domains the solvability criteria of the Dirichlet problem for an arbitrary continuous boundary function $\varphi(x)$ is the convexity of this domains. In the space of the dimension greater than two such condition is the nonnegativity of the mean curvature with respect to the outward normal for the boundary of the domain. One can find the precise formulations and the proofs of these results in works [6]-[13]. In the present paper we impose no conditions for domain $\Omega$ but we assume that for a given boundary function $\varphi(x)$ the solution to the Dirichlet problem exists. It is clear that such function $\varphi(x)$ exists for an arbitrary domain $\Omega$.

In what follows we denote by $u^{*}$ the unique solution to problem (1) with boundary data $\varphi_{i}=\varphi\left(M_{i}\right), M_{i} \in \partial \Omega$.

For arbitrary $\xi, \eta \in \mathbb{R}^{n}$ we let

$$
\delta(\xi, \eta)=\sqrt{1+|\eta|^{2}}-\sqrt{1+|\xi|^{2}}-\frac{\langle\xi, \eta-\xi\rangle}{\sqrt{1+|\xi|^{2}}} .
$$


It follows from inequality (5) that $\delta(\xi, \eta)>0$ for each $\xi \neq \eta$. Letting $\xi=\nabla f, \eta=\nabla u^{*}$ and employing equation (6), we obtain

$$
\sum_{k=1}^{N} \int_{T_{k}} \delta\left(\nabla f, \nabla u^{*}\right) d x=S\left(u^{*}\right)-S(f)+\int_{\partial \Omega} \frac{\langle\nabla f, \nu\rangle\left(u^{*}-f\right)}{\sqrt{1+|\nabla f|^{2}}} d S
$$

where $\nu$ is the outward normal to $\partial \Omega$ in the points where it exists. Employing inequality (see the proof of Theorem 1)

$$
\sqrt{1+|\eta|^{2}}-\sqrt{1+|\xi|^{2}}-\frac{\langle\xi, \eta-\xi\rangle}{\sqrt{1+|\xi|^{2}}} \geqslant \frac{|\xi-\eta|^{2}}{\sqrt{1+|\xi|^{2}}\left(\sqrt{1+|\xi|^{2}} \sqrt{1+|\eta|^{2}}+|\xi||\eta|+1\right)}
$$

we conclude that

$$
\begin{aligned}
& \sum_{k=1}^{N} \int_{T_{k}} \frac{\left|\nabla f-\nabla u^{*}\right|^{2} d x}{\sqrt{1+|\nabla f|^{2}}\left(\sqrt{1+|\nabla f|^{2}} \sqrt{1+\left|\nabla u^{*}\right|^{2}}+|\nabla f|\left|\nabla u^{*}\right|+1\right)} \\
& \quad \leqslant S\left(u^{*}\right)-S(f)+\int_{\partial \Omega} \frac{\langle\nabla f, \nu\rangle\left(u^{*}-f\right)}{\sqrt{1+|\nabla f|^{2}}} d S .
\end{aligned}
$$

We fix arbitrary $k=1, \ldots, N$. Then

$$
\begin{gathered}
\int_{T^{k}} \frac{\left|\nabla f-\nabla u^{*}\right|^{2} d x}{\sqrt{1+|\nabla f|^{2}}\left(\sqrt{1+|\nabla f|^{2}} \sqrt{1+\left|\nabla u^{*}\right|^{2}}+|\nabla f|\left|\nabla u^{*}\right|+1\right)} \\
\leqslant S\left(u^{*}\right)-S(f)+\int_{\partial \Omega} \frac{\langle\nabla f, \nu\rangle\left(u^{*}-f\right)}{\sqrt{1+|\nabla f|^{2}}} d S \equiv B .
\end{gathered}
$$

In what follows we assume that $|\nabla f| \leqslant P_{0}$ in domain $\Omega$. Then inequality (7) yields

$$
\int_{T^{k}} \frac{\left|\nabla f-\nabla u^{*}\right|^{2}}{\sqrt{1+\left|\nabla u^{*}\right|^{2}}} d x \leqslant 3\left(1+P_{0}^{2}\right) B
$$

It implies that

or

$$
\int_{T^{k}} \frac{\left|\nabla u^{*}\right|^{2}}{\sqrt{1+\left|\nabla u^{*}\right|^{2}}} d x \leqslant 3\left(1+P_{0}^{2}\right) B+2 P_{0} v\left(T^{k}\right)
$$

$$
\int_{T^{k}} \sqrt{1+\left|\nabla u^{*}\right|^{2}} d x \leqslant 3\left(1+P_{0}^{2}\right) B+\left(2 P_{0}+1\right) v\left(T^{k}\right) .
$$

Then by (8) and Hölder inequality we arrive at the estimate

$$
\int_{T^{k}}\left|\nabla f-\nabla u^{*}\right| d x \leqslant 3\left(1+P_{0}^{2}\right)\left(\left(B+v\left(T^{k}\right)\right) B\right)^{1 / 2} .
$$

Hence,

$$
\int_{T^{k}}\left|\nabla u^{*}\right| d x \leqslant P_{0} v\left(T^{k}\right)+3\left(1+P_{0}^{2}\right)\left(\left(B+v\left(T^{k}\right)\right) B\right)^{1 / 2} .
$$

Since the gradient $\nabla u^{*}$ is constant in $T_{k}$, we obtain the inequality

$$
v\left(T_{k}\right)\left|\nabla u^{*}(x)\right| \leqslant P_{0} v\left(T_{k}\right)+3\left(1+P_{0}^{2}\right)\left(\left(B+v\left(T_{k}\right)\right) B\right)^{1 / 2}, \quad x \in T_{k} .
$$

Dividing by $v\left(T_{k}\right)$, we arrive at the estimate of the gradient

$$
\left|\nabla u^{*}(x)\right| \leqslant P_{0}+3\left(1+P_{0}^{2}\right) \sqrt{\left(\alpha_{k}+1\right) \alpha_{k}},
$$


where

$$
\alpha_{k}=\frac{1}{v\left(T_{k}\right)}\left(S\left(u^{*}\right)-S(f)+\int_{\partial \Omega} \frac{\langle\nabla f, \nu\rangle\left(u^{*}-f\right)}{\sqrt{1+|\nabla f|^{2}}} d S\right) .
$$

Theorem 2. Let $f \in C^{2}(\Omega) \cap C(\bar{\Omega})$ be a solution to equation (6) such that $\left.f\right|_{\partial \Omega}=\left.\varphi\right|_{\partial \Omega}$ and $P_{0}=\sup |\nabla f|<+\infty$. Suppose that $u^{*}$ is a solution to problem (1) subject to the condition $u^{*}\left(M_{i}\right) \stackrel{\Omega}{=} \varphi\left(M_{i}\right), M_{i} \in \partial \Omega$. Then inequality (9) holds true for each point $x \in \Omega$.

Remark. Denote by $f^{L}$ the piecewise-linear function constructed by the values of functionf at points $M_{i}, i=1 \ldots, m$. If the quantity

$$
A(f)=\frac{1}{\min _{1 \leqslant k \leqslant N} v\left(T_{k}\right)}\left(S\left(f^{L}\right)-S(f)+\int_{\partial \Omega} \frac{\langle\nabla f, \nu\rangle\left(u^{*}-f\right)}{\sqrt{1+|\nabla f|^{2}}} d S\right)
$$

is bounded for sufficiently smooth functions $f$ as the fineness of partition $\mu=\max _{1 \leqslant k \leqslant N} \operatorname{diam} T_{k}$ of domain $\Omega$ tends to zero in a certain way, then by Theorem 2 and the inequality $S\left(u^{*}\right) \leqslant S\left(f^{L}\right)$ we conclude that the approximate solution $u^{*}$ has a gradient bounded by a constant independent of the partition fineness.

\section{Approximation of AREA FUnCtional}

Let us study the quantity $S\left(f^{L}\right)-S(f)$ for functions $f \in C^{3}(\Omega)$ as $n=2$, where $\Omega$ is a closed rectangle with the sides parallel to the coordinate axes. Consider the surface defined as the graph of a function $z=f(x, y)$ over set $\Omega$.

Let $\Omega=[a, b] \times[c, d]$ and $a=x_{0}<x_{1}<\cdots<x_{n}=b, c=y_{0}<y_{1}<\cdots<y_{m}=d$. We partition $\Omega$ into rectangles $\Omega_{i j}=\left[x_{i}, x_{i+1}\right] \times\left[y_{j}, y_{j+1}\right], 0 \leqslant i \leqslant n-1,0 \leqslant j \leqslant m-1$. Then we split each of these rectangles by the right or left diagonal. All the following arguments are made for the rectangle $\left[x_{0}, x_{1}\right] \times\left[y_{0}, y_{1}\right]$ since the estimate for error of calculating the area for other rectangles is similar.

If the splitting is made by the right diagonal, the area under the graph of piecewise-linear function over the diagonal is

$$
S_{U}^{r}=\frac{1}{2}\left(x_{1}-x_{0}\right)\left(y_{1}-y_{0}\right) \sqrt{1+\left(\frac{f\left(x_{1}, y_{1}\right)-f\left(x_{0}, y_{1}\right)}{x_{1}-x_{0}}\right)^{2}+\left(\frac{f\left(x_{0}, y_{1}\right)-f\left(x_{0}, y_{0}\right)}{y_{1}-y_{0}}\right)^{2}},
$$

and for the triangle under the diagonal

$$
S_{D}^{l}=\frac{1}{2}\left(x_{1}-x_{0}\right)\left(y_{1}-y_{0}\right) \sqrt{1+\left(\frac{f\left(x_{1}, y_{0}\right)-f\left(x_{0}, y_{0}\right)}{x_{1}-x_{0}}\right)^{2}+\left(\frac{f\left(x_{0}, y_{1}\right)-f\left(x_{1}, y_{1}\right)}{y_{1}-y_{0}}\right)^{2}} .
$$

If the triangle is split by the left diagonal, the area under the graph of the piecewise-linear function over the diagonal equals

$$
S_{U}^{l}=\frac{1}{2}\left(x_{1}-x_{0}\right)\left(y_{1}-y_{0}\right) \sqrt{1+\left(\frac{f\left(x_{0}, y_{1}\right)-f\left(x_{1}, y_{1}\right)}{x_{1}-x_{0}}\right)^{2}+\left(\frac{f\left(x_{1}, y_{0}\right)-f\left(x_{1}, y_{1}\right)}{y_{1}-y_{0}}\right)^{2}},
$$

and for the triangle under the diagonal

$$
S_{D}^{l}=\frac{1}{2}\left(x_{1}-x_{0}\right)\left(y_{1}-y_{0}\right) \sqrt{1+\left(\frac{f\left(x_{1}, y_{0}\right)-f\left(x_{0}, y_{1}\right)}{x_{1}-x_{0}}\right)^{2}+\left(\frac{f\left(x_{0}, y_{1}\right)-f\left(x_{0}, y_{0}\right)}{y_{1}-y_{0}}\right)^{2}} .
$$

We denote by $S$ the area of the obtained piecewise-linear surface. We have 
Theorem 3. Let $f(x, y) \in C^{3}(\Omega)$ and

$$
\begin{gathered}
M_{2}=\sup _{\Omega} \max \left\{\left|f_{x x}^{\prime \prime}\right|,\left|f_{x y}^{\prime \prime}\right|,\left|f_{y y}^{\prime \prime}\right|\right\}, \quad M_{3}=\sup _{\Omega} \max \left\{\left|f_{x x x}^{\prime \prime \prime}\right|,\left|f_{x x y}^{\prime \prime \prime}\right|,\left|f_{x y y}^{\prime \prime \prime}\right|,\left|f_{y y y}^{\prime \prime \prime}\right|\right\}, \\
h_{1}=\max _{1 \leqslant i \leqslant n}\left(x_{i}-x_{i-1}\right), \quad h_{2}=\max _{1 \leqslant j \leqslant m}\left(y_{j}-y_{j-1}\right), \quad h=\max \left(h_{1}, h_{2}\right) .
\end{gathered}
$$

Then

$$
\begin{aligned}
& \left|\iint_{\Omega} \sqrt{1+|\nabla f(x, y)|^{2}} d x d y-S\right| \\
& \leqslant|\Omega|\left(2 M_{3}+12\left(3+\frac{h}{\min \left\{h_{1}, h_{2}\right\}}\right) M_{2}^{2}+\frac{5}{2} M_{3}^{2} h^{2}\right) h^{2} .
\end{aligned}
$$

Proof. For the sake of brevity we assume that the rectangles in the partition of set $\Omega$ are split by the right diagonal. The results for this case and for the general case coincide.

Consider the rectangle $\left[x_{0}, x_{1}\right] \times\left[y_{0}, y_{1}\right]$. By means of Newton interpolation formula (see [14, Ch. 3, Sect. 12]) we obtain a linear approximation $l_{U}$ and $l_{D}$ for function $f(x, y)$ over upper and lower partition triangles of this rectangle

$$
\begin{aligned}
f(x, y)= & l_{U}(x, y)+R_{U}(x, y)=f\left(x_{0}, y_{0}\right)+\left(x-x_{0}\right) f\left(x_{0} ; x_{1}, y_{0}\right) \\
& +\left(y-y_{0}\right) f\left(x_{0}, y_{0} ; y_{1}\right)+\left(y-y_{0}\right)\left(y-y_{1}\right) f\left(x, y_{0} ; y_{1} ; y\right) \\
& +\left(x-x_{0}\right)\left(y-y_{1}\right) f\left(x_{0} ; x, y_{0} ; y_{1}\right)+\left(x-x_{0}\right)\left(x-x_{1}\right) f\left(x_{0} ; x_{1} ; x, y_{0}\right), \\
f(x, y)= & l_{D}(x, y)+R_{D}(x, y)=f\left(x_{1}, y_{1}\right)+\left(x-x_{1}\right) f\left(x_{1} ; x_{0}, y_{1}\right) \\
& +\left(y-y_{1}\right) f\left(x_{1}, y_{1} ; y_{0}\right)+\left(y-y_{1}\right)\left(y-y_{0}\right) f\left(x, y ; y_{1} ; y_{0}\right) \\
& +\left(x-x_{1}\right)\left(y-y_{0}\right) f\left(x_{0} ; x, y_{1} ; y_{0}\right)+\left(x-x_{0}\right)\left(x-x_{1}\right) f\left(x_{0} ; x_{1} ; x, y_{1}\right),
\end{aligned}
$$

where $f\left(\alpha_{1} ; \alpha_{2} ; \alpha_{3} ; \cdots ; \alpha_{n}, \beta_{1} ; \beta_{2} ; \beta_{3} ; \cdots ; \beta_{m}\right)$ are separated differences of function $f(x, y)$ (see [14, Ch. 2, Sect. 5]). In particular,

Then

$$
\begin{array}{ll}
f\left(x_{0} ; x_{1}, y_{0}\right)=\frac{f\left(x_{1}, y_{0}\right)-f\left(x_{0}, y_{0}\right)}{x_{1}-x_{0}}, & f\left(x_{0}, y_{0} ; y_{1}\right)=\frac{f\left(x_{0}, y_{1}\right)-f\left(x_{0}, y_{0}\right)}{y_{1}-y_{0}}, \\
f\left(x_{0} ; x_{1}, y_{1}\right)=\frac{f\left(x_{1}, y_{1}\right)-f\left(x_{0}, y_{1}\right)}{x_{1}-x_{0}}, & f\left(x_{1} ; y_{0}, y_{1}\right)=\frac{f\left(x_{1}, y_{1}\right)-f\left(x_{1}, y_{0}\right)}{y_{1}-y_{0}} .
\end{array}
$$

$$
\begin{aligned}
& \frac{\partial f}{\partial x}(x, y)=\frac{f\left(x_{1}, y_{0}\right)-f\left(x_{0}, y_{0}\right)}{x_{1}-x_{0}}+\frac{\partial R_{U}}{\partial x}(x, y), \\
& \frac{\partial f}{\partial y}(x, y)=\frac{f\left(x_{0}, y_{1}\right)-f\left(x_{0}, y_{0}\right)}{y_{1}-y_{0}}+\frac{\partial R_{U}}{\partial y}(x, y), \\
& \frac{\partial f}{\partial x}(x, y)=\frac{f\left(x_{1}, y_{1}\right)-f\left(x_{0}, y_{1}\right)}{x_{1}-x_{0}}+\frac{\partial R_{D}}{\partial x}(x, y), \\
& \frac{\partial f}{\partial y}(x, y)=\frac{f\left(x_{1}, y_{1}\right)-f\left(x_{1}, y_{0}\right)}{y_{1}-y_{0}}+\frac{\partial R_{D}}{\partial y}(x, y) .
\end{aligned}
$$

Consider the difference of areas under the graph of the given function and the graph of the obtained interpolation by the linear function over the upper partition triangle

$$
\begin{aligned}
& \iint_{U} \sqrt{1+|\nabla f|^{2}} d x d y-S_{U}^{r}=\iint_{U} \sqrt{1+|\nabla f|^{2}} d x d y \\
& -\iint_{U} \sqrt{1+\left(\frac{f\left(x_{1}, y_{1}\right)-f\left(x_{0}, y_{1}\right)}{x_{1}-x_{0}}\right)^{2}+\left(\frac{f\left(x_{0}, y_{1}\right)-f\left(x_{0}, y_{0}\right)}{y_{1}-y_{0}}\right)^{2}} d x d y
\end{aligned}
$$




$$
\begin{gathered}
=\iint_{U} \sqrt{1+|\nabla f|^{2}} d x d y-\iint_{U} \sqrt{1+\left(\frac{\partial f}{\partial x}(x, y)-\frac{\partial R_{U}}{\partial x}(x, y)\right)^{2}+\left(\frac{\partial f}{\partial y}(x, y)-\frac{\partial R_{U}}{\partial y}(x, y)\right)^{2}} \\
=\iint_{U} C_{U}(x, y)\left(2 \frac{\partial f}{\partial x}(x, y) \frac{\partial R_{U}}{\partial x}(x, y)+2 \frac{\partial f}{\partial y}(x, y) \frac{\partial R_{U}}{\partial y}(x, y)\right. \\
\left.-\left(\frac{\partial R_{U}}{\partial x}(x, y)\right)^{2}-\left(\frac{\partial R_{U}}{\partial y}(x, y)\right)^{2}\right) d x d y
\end{gathered}
$$

where

$$
C_{U}(x, y)=\left(\sqrt{1+|\nabla f|^{2}}+\sqrt{1+\left(\frac{\partial f}{\partial x}-\frac{\partial R_{U}}{\partial x}\right)^{2}+\left(\frac{\partial f}{\partial y}-\frac{\partial R_{U}}{\partial y}\right)^{2}}\right)^{-1}
$$

Let $y=\Gamma_{1}(x)$ define the hypotenuse of the partition triangle and $x=\Gamma_{2}(y)$ be the inverse function. We integrate by parts in the integral in the right hand side of the identity

$$
\begin{aligned}
\iint_{U} & \sqrt{1+|\nabla f|^{2}} d x d y-S_{U}^{r}=2 \int_{y_{0}}^{y_{1}}\left(\left.R_{U}(x, y) \frac{\partial f}{\partial x}(x, y) C_{U}(x, y)\right|_{x=x_{0}} ^{x=\Gamma_{2}(y)}\right. \\
& \left.-\int_{x_{0}}^{\Gamma_{2}(y)} R_{U}(x, y)\left[\frac{\partial f}{\partial x}(x, y) \frac{\partial C_{U}}{\partial x}(x, y)+\frac{\partial^{2} f}{\partial x^{2}}(x, y) C_{U}(x, y)\right] d x\right) d y \\
& +2 \int_{x_{0}}^{x_{1}}\left(\left.R_{U}(x, y) \frac{\partial f}{\partial y}(x, y) C_{U}(x, y)\right|_{y=\Gamma_{1}(x)} ^{y=y_{1}}\right. \\
& \left.-\int_{\Gamma_{1}(x)}^{y_{1}} R_{U}(x, y)\left[\frac{\partial f}{\partial y}(x, y) \frac{\partial C_{U}}{\partial y}(x, y)+\frac{\partial^{2} f}{\partial y^{2}}(x, y) C_{U}(x, y)\right] d y\right) d x-\iint_{U}\left|\nabla R_{U}(x, y)\right|^{2} d x d y
\end{aligned}
$$

We indicate by $C_{D}(x, y)$ the function on $D$ similar to function $C_{U}(x, y)$. In the same way on $D$ we obtain the identity

$$
\begin{aligned}
\iint_{D} & \sqrt{1+|\nabla f|^{2}} d x d y-S_{D}^{r}=2 \int_{y_{0}}^{y_{1}}\left(\left.R_{D}(x, y) \frac{\partial f}{\partial x}(x, y) C_{D}(x, y)\right|_{x=\Gamma_{2}(y)} ^{x=x_{1}}\right. \\
& \left.-\int_{\Gamma_{2}(y)}^{x_{1}} R_{D}(x, y)\left[\frac{\partial f}{\partial x}(x, y) \frac{\partial}{\partial C_{D} x}(x, y)+\frac{\partial^{2} f}{\partial x^{2}}(x, y) C_{D}(x, y)\right] d x\right) d y \\
& +2 \int_{x_{0}}^{x_{1}}\left(\left.R_{D}(x, y) \frac{\partial f}{\partial y}(x, y) C_{D}(x, y)\right|_{y=y_{0}} ^{y=\Gamma_{1}(x)}\right. \\
& \left.-\int_{y=y_{0}}^{\Gamma_{1}(x)} R_{D}(x, y)\left[\frac{\partial f}{\partial y}(x, y) \frac{\partial C_{D}}{\partial y}(x, y)+\frac{\partial^{2} f}{\partial y^{2}}(x, y) C_{D}(x, y)\right] d y\right) d x-\iint_{D}\left|\nabla R_{D}(x, y)\right|^{2} d x d y .
\end{aligned}
$$


We sum up the obtained identities taking into account that on $\Gamma$ the values of continuous functions coincide

$$
\begin{aligned}
\iint_{U \cup D} \sqrt{1+|\nabla f|^{2}} & d x d y-\left(S_{U}^{r}+S_{D}^{r}\right) \\
= & 2 \int_{x_{0}}^{x_{1}}\left(R_{U}\left(x, y_{1}\right) f_{y}^{\prime}\left(x, y_{1}\right) C_{U}\left(x, y_{1}\right)-R_{D}\left(x, y_{0}\right) f_{y}^{\prime}\left(x, y_{0}\right) C_{D}\left(x, y_{0}\right)\right) d x \\
& +2 \int_{y_{0}}^{y_{1}}\left(R_{D}\left(x_{1}, y\right) f_{x}^{\prime}\left(x_{1}, y\right) C_{D}\left(x_{1}, y\right)-R_{U}\left(x_{0}, y\right) f_{x}^{\prime}\left(x_{0}, y\right) C_{U}\left(x_{0}, y\right)\right) d y \\
& +2 \int_{x_{0}}^{x_{1}} R_{U}\left(\Gamma_{1}(x)\right) f_{y}^{\prime}\left(\Gamma_{1}(x)\right)\left(C_{U}\left(\Gamma_{1}(x)\right)-C_{D}\left(\Gamma_{1}(x)\right)\right) d x \\
& -2 \int_{y_{0}}^{y_{1}} R_{U}\left(\Gamma_{2}(y)\right) f_{x}^{\prime}\left(\Gamma_{2}(y)\right)\left(C_{D}\left(\Gamma_{2}(y)\right)-C_{U}\left(\Gamma_{2}(y)\right)\right) d y \\
& -2 \iint_{U}\left(R_{U} \operatorname{div}\left(C_{U}(x, y) \nabla f(x, y)\right)+\frac{1}{2}\left|\nabla R_{U}(x, y)\right|^{2}\right) d x d y \\
& -2 \iint_{D}\left(R_{D} \operatorname{div}\left(C_{D}(x, y) \nabla f(x, y)\right)+\frac{1}{2}\left|\nabla R_{D}(x, y)\right|^{2}\right) d x d y .
\end{aligned}
$$

It is easy to make sure that

$$
\begin{aligned}
& \left|C_{U}(x, y)-C_{D}(x, y)\right| \leqslant 2 C_{U}(x, y) C_{D}(x, y) M_{2} h \\
& \left|C_{U}\left(x_{1}, y\right)-C_{D}\left(x_{0}, y\right)\right| \leqslant 4 C_{U}(x, y) C_{D}(x, y) M_{2} h_{1}, \\
& \left|C_{U}\left(x, y_{1}\right)-C_{D}\left(x, y_{0}\right)\right| \leqslant 4 C_{U}(x, y) C_{D}(x, y) M_{2} h_{2} .
\end{aligned}
$$

Then, employing that the separated differences are equal to the values of the derivatives at some point of the domain, we obtain

$$
\begin{aligned}
& \left|\int_{x_{0}}^{x_{1}}\left(R_{U}\left(x, y_{1}\right) \frac{\partial f}{\partial y}\left(x, y_{1}\right) C_{U}\left(x, y_{1}\right)-R_{D}\left(x, y_{0}\right) \frac{\partial f}{\partial y}\left(x, y_{0}\right) C_{D}\left(x, y_{0}\right)\right) d x\right| \\
& =\mid \int_{x_{0}}^{x_{1}}\left(x-x_{0}\right)\left(x-x_{1}\right)\left[\frac{\partial f}{\partial y}\left(x, y_{1}\right) C_{U}\left(x, y_{1}\right) \frac{\partial^{3} f}{\partial x^{2} \partial y}\left(\xi_{1}, \eta_{1}\right)\left(y_{1}-y_{0}\right)\right. \\
& \quad+\frac{\partial^{2} f}{\partial y^{2}}\left(\xi_{2}, \eta_{2}\right) C_{U}\left(x, y_{1}\right) \frac{\partial^{2} f}{\partial x^{2}}\left(\xi_{3}, y_{0}\right)\left(y_{1}-y_{0}\right) \\
& \left.\quad+\frac{\partial^{2} f}{\partial x^{2}}\left(\xi_{3}, y_{0}\right) f_{y}\left(x, y_{0}\right)\left(C_{U}\left(x, y_{1}\right)-C_{D}\left(x, y_{0}\right)\right)\right] d x \mid \leqslant \frac{1}{4} h_{1} h_{2}\left(M_{3}+5 M_{2}^{2}\right) h_{1}^{2} .
\end{aligned}
$$


In the same way,

$$
\begin{aligned}
\left|\int_{y_{0}}^{y_{1}}\left(R_{D}\left(x_{1}, y\right) f_{x}\left(x_{1}, y\right) C_{U}\left(x_{1}, y\right)-R_{U}\left(x_{0}, y\right) f_{x}\left(x_{0}, y\right) C_{U}\left(x_{0}, y\right)\right) d y\right| \\
\leqslant \frac{1}{4} h_{1} h_{2}\left(M_{3}+5 M_{2}^{2}\right) h_{2}^{2} .
\end{aligned}
$$

Let us estimate the modules of interpolation error for each of the triangles:

$$
\left|R_{U}(x, y)\right| \leqslant M_{2}\left(\frac{1}{4} h_{2}^{2}+h_{1} h_{2}+\frac{1}{4} h_{1}^{2}\right), \quad\left|R_{D}(x, y)\right| \leqslant M_{2}\left(\frac{1}{4} h_{2}^{2}+h_{1} h_{2}+\frac{1}{4} h_{1}^{2}\right) .
$$

These inequalities can be employed as the estimates for the errors $R_{U}(\Gamma)$ and $R_{D}(\Gamma)$ on diagonal $\Gamma$

$$
y=y_{0}+\frac{y_{1}-y_{0}}{x_{1}-x_{0}}\left(x-x_{0}\right)
$$

of the considered rectangle. Then the relations (11) yield

$$
\begin{aligned}
& \left|\int_{x_{0}}^{x_{1}} R_{U}\left(\Gamma_{1}(x)\right) f_{y}^{\prime}\left(\Gamma_{1}(x)\right)\left(C_{U}\left(\Gamma_{1}(x)\right)-C_{D}\left(\Gamma_{1}(x)\right)\right) d x\right| \leqslant 2 M_{2}^{2}\left(\frac{1}{4} h_{2}^{2}+h_{1} h_{2}+\frac{1}{4} h_{1}^{2}\right) h_{1} h, \\
& \left|\int_{y_{0}}^{y_{1}} R_{U}\left(\Gamma_{2}(y)\right) f_{x}^{\prime}\left(\Gamma_{2}(y)\right)\left(C_{D}\left(\Gamma_{2}(y)\right)-C_{U}\left(\Gamma_{2}(y)\right)\right) d y\right| \leqslant 2 M_{2}^{2}\left(\frac{1}{4} h_{2}^{2}+h_{1} h_{2}+\frac{1}{4} h_{1}^{2}\right) h_{2} h .
\end{aligned}
$$

Let us estimates the squares of moduli of the errors gradient

$$
\begin{aligned}
\frac{\partial R_{U}}{\partial x}= & \left(y-y_{0}\right)\left(y-y_{1}\right) f\left(x ; x, y_{0} ; y_{1} ; y\right)+\left(y-y_{1}\right) f\left(x_{0} ; x, y_{0} ; y_{1}\right) \\
& +\left(x-x_{0}\right)\left(y-y_{1}\right) f\left(x_{0} ; x ; x, y_{0} ; y_{1}\right)+\left(x-x_{0}\right)\left(x-x_{1}\right) f\left(x_{0} ; x_{1} ; x ; x, y_{0}\right) \\
& +2\left(x-\frac{x_{0}+x_{1}}{2}\right) f\left(x_{0} ; x_{1} ; x, y_{0}\right), \\
\frac{\partial R_{U}}{\partial y}= & \left(y-y_{0}\right)\left(y-y_{1}\right) f\left(x, y_{0} ; y_{1} ; y ; y\right)+2\left(y-\frac{y_{0}+y_{1}}{2}\right) f\left(x, y_{0} ; y_{1} ; y\right) \\
& +\left(x-x_{0}\right) f\left(x_{0} ; x, y_{0} ; y_{1}\right), \\
\frac{\partial R_{D}}{\partial x}= & \left(y-y_{1}\right)\left(y-y_{0}\right) f\left(x ; x, y_{1} ; y_{0} ; y\right)+\left(y-y_{1}\right) f\left(x_{0} ; x, y_{1} ; y_{0}\right) \\
& +\left(x-x_{0}\right)\left(x-x_{1}\right) f\left(x_{0} ; x_{1} ; x ; x, y_{1}\right)+2\left(x-\frac{x_{0}+x_{1}}{2}\right) f\left(x_{0} ; x_{1} ; x ; x, y_{1}\right) \\
& +\left(x-x_{1}\right)\left(y-y_{1}\right) f\left(x_{0} ; x ; x, y_{1} ; y_{0}\right), \\
\frac{\partial R_{D}}{\partial y}= & \left(y-y_{1}\right)\left(y-y_{0}\right) f\left(x, y ; y ; y_{1} ; y_{0}\right)+2\left(y-\frac{y_{0}+y_{1}}{2}\right) f\left(x, y ; y_{1} ; y_{0}\right) \\
& +\left(x-x_{1}\right) f\left(x_{0} ; x, y_{1} ; y_{0}\right) .
\end{aligned}
$$

By Cauchy-Schwarz inequality it implies

$$
\begin{aligned}
& \left|\frac{\partial R_{U}}{\partial x}\right|^{2} \leqslant \frac{1}{16} M_{3}^{2} h_{2}^{4}+M_{2}^{2} h_{2}^{2}+M_{3}^{2} h_{1}^{2} h_{2}^{2}+\frac{1}{16} M_{3}^{2} h_{1}^{4}+M_{2}^{2} h_{1}^{2} \\
& \left|\frac{\partial R_{U}}{\partial y}\right|^{2} \leqslant \frac{1}{16} M_{3}^{2} h_{2}^{4}+M_{2}^{2} h_{2}^{2}+M_{2}^{2} h_{1}^{2},
\end{aligned}
$$




$$
\left|\nabla R_{U}\right|^{2} \leqslant \frac{1}{8} M_{3}^{2} h_{2}^{4}+2 M_{2}^{2} h_{2}^{2}+M_{3}^{2} h_{1}^{2} h_{2}^{2}+\frac{1}{16} M_{3}^{2} h_{1}^{2}+2 M_{2}^{2} h_{1}^{2} .
$$

In the same way,

$$
\left|\nabla R_{D}\right|^{2} \leqslant \frac{1}{8} M_{3}^{2} h_{2}^{4}+2 M_{2}^{2} h_{2}^{2}+M_{3}^{2} h_{1}^{2} h_{2}^{2}+\frac{1}{16} M_{3}^{2} h_{1}^{2}+2 M_{2}^{2} h_{1}^{2} .
$$

Then we observe that $U$ satisfies

$$
\begin{aligned}
& \operatorname{div}\left(C_{U} \nabla f\right)=C_{U} \Delta f+\left\langle\nabla C_{U}, \nabla f\right\rangle, \\
& \frac{\partial C_{U}}{\partial x}=-\left(C_{U}\right)^{2} \frac{\partial}{\partial x} \sqrt{1+|\nabla f|^{2}}=-\left(C_{U}\right)^{2} \frac{f_{x}^{\prime} f_{x x}^{\prime \prime}+f_{y}^{\prime} f_{x y}^{\prime \prime}}{\sqrt{1+|\nabla f|^{2}}}, \\
& \frac{\partial C_{U}}{\partial y}=-\left(C_{U}\right)^{2} \frac{\partial}{\partial y} \sqrt{1+|\nabla f|^{2}}=-\left(C_{U}\right)^{2} \frac{f_{x}^{\prime} f_{x y}^{\prime \prime}+f_{y}^{\prime} f_{y y}^{\prime \prime}}{\sqrt{1+|\nabla f|^{2}}}
\end{aligned}
$$

Hence,

And since

$$
\left|\left\langle\nabla f, \nabla C_{U}\right\rangle\right|=\left|\frac{C_{U}^{2}}{\sqrt{1+|\nabla f|^{2}}}\left(\left(f_{x}^{\prime}\right)^{2} f_{x x}^{\prime \prime}+2 f_{x}^{\prime} f_{y}^{\prime} f_{x y}^{\prime \prime}+\left(f_{y}^{\prime}\right)^{2} f_{y y}^{\prime \prime}\right)\right| \leqslant 4 M_{2} .
$$

$$
\left|C_{U} \Delta f\right| \leqslant 2 M_{2}
$$

then

$$
\left|\operatorname{div}\left(C_{U} \nabla f\right)\right| \leqslant 6 M_{2}
$$

In the same way for $D$ we have

$$
\left|\operatorname{div}\left(C_{D} \nabla f\right)\right| \leqslant 6 M_{2} .
$$

Let us estimate the modulus of the sum in the right hand side of identity 10 . Employing inequalities (14), (17)-20), we obtain

$$
\begin{aligned}
& \mid \iint_{U}\left(R_{U} \operatorname{div}\left(C_{U}(x, y) \nabla f(x, y)\right)+\frac{1}{2}\left|\nabla R_{U}(x, y)\right|^{2}\right) d x d y \\
& \quad+\iint_{D}\left(R_{D} \operatorname{div}\left(C_{D}(x, y) \nabla f(x, y)\right)+\frac{1}{2}\left|\nabla R_{D}(x, y)\right|^{2}\right) d x d y \mid \\
& \leqslant\left(3 M_{2}^{2}\left(h_{2}^{2}+4 h_{1} h_{2}+h_{1}^{2}\right)+\frac{1}{8} M_{3}^{2} h_{2}^{4}+2 M_{2}^{2} h_{2}^{2}+M_{3}^{2} h_{1}^{2} h_{2}^{2}+\frac{1}{16} M_{3}^{2} h_{1}^{2}+2 M_{2}^{2} h_{1}^{2}\right)|P| \\
& \leqslant\left(\frac{1}{8} M_{3}^{2} h_{2}^{4}+5 M_{2}^{2} h_{2}^{2}+3 M_{2}^{2} h_{1} h_{2}+M_{3}^{2} h_{1}^{2} h_{2}^{2}+5 M_{2}^{2} h_{1}^{2}+\frac{1}{16} M_{3}^{2} h_{1}^{4}\right)|P|,
\end{aligned}
$$

where $|P|$ is the area of the rectangle $P=\left[x_{0}, x_{1}\right] \times\left[y_{0}, y_{1}\right]$. Now by (10), (12), (13), (15), (16), (21), and the fact that the area of rectangle $P$ does not exceeds $h_{1} h_{2}$, we get

$$
\begin{aligned}
& \left|\iint_{P} \sqrt{1+|\nabla f|^{2}} d x d y-\left(S_{U}^{r}+S_{D}^{r}\right)\right| \leqslant \frac{1}{2} h_{1} h_{2}\left(M_{3}+5 M_{2}^{2}\right)\left(h_{1}^{2}+h_{2}^{2}\right) \\
& +4 M_{2}^{2}\left(\frac{1}{4} h_{2}^{2}+h_{1} h_{2}+\frac{1}{4} h_{1}^{2}\right) h\left(h_{1}+h_{2}\right) \\
& \quad+\left(\frac{1}{8} M_{3}^{2} h_{2}^{4}+5 M_{2}^{2} h_{2}^{2}+3 M_{2}^{2} h_{1} h_{2}+M_{3}^{2} h_{1}^{2} h_{2}^{2}+5 M_{2}^{2} h_{1}^{2}+\frac{1}{16} M_{3}^{2} h_{1}^{4}\right) h_{1} h_{2}
\end{aligned}
$$




$$
\leqslant h_{1} h_{2}\left(2 M_{3} h^{2}+\left(36+12 \frac{h}{\min \left\{h_{1}, h_{2}\right\}}\right) M_{2}^{2} h^{2}+\frac{5}{2} M_{3}^{2} h^{4}\right) .
$$

Summing up the inequality over all the partition rectangles for set $\Omega$, we finally obtain

$$
\left|\iint_{\Omega} \sqrt{1+|\nabla f|^{2}} d x d y-S\right| \leqslant|\Omega|\left(2 M_{3}+12\left(3+\frac{h}{\min \left\{h_{1}, h_{2}\right\}}\right) M_{2}^{2}+\frac{5}{2} M_{3}^{2} h^{2}\right) h^{2} .
$$

\section{UNIFORM CONVERGENCE OF APPROXIMATE SOLUTIONS}

Now we are going to obtain the uniform estimate for piecewise-linear solutions of minimal surface equation. Let $f$ be a solution to equation (6) in domain $\Omega$. We assume that

$$
\sup _{\Omega}|\nabla f|=P_{0}<+\infty .
$$

In what follows we shall argue as in works [15], [16]. We denote by $f^{L}$ the piecewise-linear function such that $f^{L}\left(M_{i}\right)=f\left(M_{i}\right)$. We let $f^{t}(x)=u^{*}(x)+t\left(f^{L}(x)-u^{*}(x)\right)$ and $P_{1}=\sup _{\Omega}\left|\nabla u^{*}\right|$, $P=\max \left\{1, P_{0}, P_{1}\right\}$. It is clear that $\left.u^{*}\right|_{\partial \Omega}=\left.f^{L}\right|_{\partial \Omega}$. For each $t \in \mathbb{R}$ function $f^{t}(x)$ is piecewiselinear and one can calculate the area under its graph

$$
\sigma(t)=\int_{\Omega} \sqrt{1+\left|\nabla f^{t}\right|^{2}} d x
$$

Since as $t=0$ function $\sigma(t)$ achieves its minimum, then $\sigma^{\prime}(0)=0$. Employing this identity, we obtain

$$
\begin{aligned}
S\left(f^{L}\right)-S\left(u^{*}\right) & =\int_{0}^{1} d s \int_{0}^{s} \sigma^{\prime \prime}(t) d t \\
& =\int_{0}^{1} d s \int_{0}^{s} d t \int_{\Omega} \frac{\left(1+\left|\nabla f^{t}\right|^{2}\right)\left|\nabla f^{L}-\nabla u^{*}\right|^{2}-\left\langle\nabla f^{t}, \nabla f^{L}-\nabla u^{*}\right\rangle^{2}}{\left(1+\left|\nabla f^{t}\right|^{2}\right)^{3 / 2}} d x \\
& \geqslant \int_{0}^{1} d s \int_{0}^{s} d t \int_{\Omega} \frac{\left|\nabla f^{L}-\nabla u^{*}\right|^{2}}{\left(1+\left|\nabla f^{t}\right|^{2}\right)^{3 / 2}} d x \geqslant \frac{1}{\sqrt{\left(1+P^{2}\right)^{3}}} \int_{\Omega}\left|\nabla f^{L}-\nabla u^{*}\right|^{2} d x .
\end{aligned}
$$

We employ Poincaré inequality (see, for instance, [17, Sect. 7.8]) for function $h(x)=f^{L}(x)-$ $u^{*}(x),\left.h\right|_{\partial \Omega}=0$. By $(22)$ we get

$$
S\left(f^{L}\right)-S\left(u^{*}\right) \geqslant \frac{\lambda(\Omega)}{\sqrt{\left(1+P^{2}\right)^{3}}} \int_{\Omega}|h(x)|^{2} d x,
$$

where $\lambda(\Omega)=\left(\omega_{n} /|\Omega|\right)^{2 / n}$ and $\omega_{n},|\Omega|$ are $n$-dimensional volumes of the unit ball and domain $\Omega$, respectively. Then we let $M=\sup _{\Omega}|h|$ and without loss of generality we can assume that there exists a point $x_{0} \in \Omega$ satisfying $h\left(x_{0}\right)=M$. Let us show that $B_{M / 4 P}\left(x_{0}\right) \subset \Omega$. Indeed, let $x^{\prime} \in \partial \Omega$ be such that $\left|x_{0}-x^{\prime}\right|=\operatorname{dist}(x, \partial \Omega)$. Hence,

$$
2 P\left|x_{0}-x^{\prime}\right| \geqslant h\left(x_{0}\right)-h\left(x^{\prime}\right)=M-h\left(x^{\prime}\right) \geqslant M-M^{\prime} \geqslant M / 2 .
$$


Thus, the distance from point $x_{0}$ to boundary $\partial \Omega$ is greater than $M / 4 P$. Therefore, $B_{M / 4 P}\left(x_{0}\right) \subset \Omega$. Suppose that $x \in B_{M / 4 P}\left(x_{0}\right)$. Then

$$
h(x) \geqslant h\left(x_{0}\right)-2 P\left|x-x_{0}\right|>M-2 P \frac{M}{4 P}=M / 2 .
$$

Hence, $B_{M / 4 P}\left(x_{0}\right) \subset D_{M}$, where

$$
D_{M}=\{x \in \Omega:|h|>M / 2\} \subset \subset \Omega .
$$

Therefore,

$$
\int_{\Omega}|h(x)|^{2} d x \geqslant \int_{D_{M}}|h|^{2} d x \geqslant \int_{B_{M / 4 P}\left(x_{0}\right)}\left(\frac{M}{2}\right)^{2} d x=\frac{M^{2}}{4}\left(\frac{M}{4 P}\right)^{n} \omega_{n}=\frac{M^{n+2}}{4^{n+1} P^{n}} \omega_{n} .
$$

Hence,

$$
\max _{\Omega}\left|f^{L}-u^{*}\right| \leqslant 4 P^{4 / 3}\left(\frac{S\left(f^{L}\right)-S\left(u^{*}\right)}{\lambda(\Omega) \omega_{n}}\right)^{\frac{1}{n+2}} .
$$

Theorem 4. Let $f \in C^{2}(\Omega) \cap C(\bar{\Omega})$ be a solution to minimal surface equation (6) and $u^{*}$ be a piecewise-linear function solving problem (1) with $\varphi_{i}=f\left(M_{i}\right)$ for each $M_{i} \in \partial \Omega$. Suppose that $P_{0}=\sup _{\Omega}|\nabla f|<+\infty$ and $P_{1}=\sup _{\Omega}\left|\nabla u^{*}\right|$. Then

$$
\max _{\Omega}\left|f^{L}-u^{*}\right| \leqslant 4 P^{4 / 3}\left(\frac{S\left(f^{L}\right)-S\left(u^{*}\right)}{\lambda(\Omega) \omega_{n}}\right)^{\frac{1}{n+2}}
$$

where $P=\max \left\{1, P_{0}, P_{1}\right\}$.

Now let $\Omega$ be a rectangle $[a, b] \times[c, d]$. We fix a natural number $m$ and consider the partition of the rectangle defined by the points $x_{i}=a+\frac{i}{m}(b-a), y_{j}=c+\frac{j}{m}(d-c), i, j=0,1, \ldots, m$. We partition each of the rectangles $\left[x_{i}, x_{i+1}\right] \times\left[y_{j}, y_{j+1}\right], i, j=0,1, \ldots, m-1$ by the diagonal connecting vertices $\left(x_{i}, y_{j}\right)$ and $\left(x_{i+1}, y_{j+1}\right)$ into two triangles. Suppose in rectangle $\Omega$ we are given a solution $f$ to the minimal surface equation, $f \in C^{3}(\bar{\Omega})$. Let $u_{m}^{*}$ be the solution to problem (1) associated with this partition and satisfying the boundary conditions

$$
\begin{aligned}
& u_{m}^{*}\left(x_{i}, c\right)=f\left(x_{i}, c\right), u_{m}^{*}\left(x_{i}, d\right)=f\left(x_{i}, d\right), \quad i=0, \ldots, m, \\
& u_{m}^{*}\left(a, y_{j}\right)=f\left(a, y_{j}\right), u_{m}^{*}\left(b, y_{j}\right)=f\left(b, y_{j}\right), \quad j=0, \ldots, m .
\end{aligned}
$$

Corollary. Sequence $u_{m}^{*}$ converges uniformly in $\Omega$ to solution $f$, at that

$$
\sup _{\Omega}\left|f(x, y)-u_{m}^{*}(x, y)\right|=O\left(\frac{1}{\sqrt{m}}\right)
$$

as $m \rightarrow \infty$.

Proof. We denote by $f^{L}$ the piecewise-linear function such that $f^{L}\left(x_{i}, y_{j}\right)=f\left(x_{i}, y_{j}\right), i, j=$ $0, \ldots, m$. First let us show that the gradients of functions $u_{m}^{*}$ are bounded by a constant independent of $m$. In order to do it, we employ inequality (9). It follows from Theorem 3 that for some constant $C_{1}$ independent of $m$ we have

$$
\left|S(f)-S\left(f^{L}\right)\right| \leqslant \frac{C_{1}}{m^{2}}
$$

and applying the trapezium formula of numerical integration (see [14, Ch. 3]), we obtain

$$
\left|\int_{\partial \Omega} \frac{\langle\nabla f, \nu\rangle\left(u_{m}^{*}-f\right)}{\sqrt{1+|\nabla f|^{2}}} d S\right| \leqslant \frac{C_{2}}{m^{2}}
$$


Thus,

$$
|A(f)| \leqslant \frac{2}{(b-a)(d-c)}\left(C_{1}+C_{2}\right) \equiv C_{3} .
$$

If $P_{0}=\sup _{\Omega}|\nabla f|$, it follows from inequality $(9)$ that

$$
\left|\nabla u_{m}^{*}\right| \leqslant P_{0}+3\left(1+P_{0}^{2}\right) \sqrt{C_{3}\left(1+C_{3}\right)} \equiv P_{1} .
$$

Let us estimate the quantity $S\left(f^{L}\right)-S\left(u_{m}^{*}\right)$. We construct arbitrary function $\tilde{u}_{m}$ such that $\tilde{u}_{m}=u_{m}^{*}$ in rectangle $\Omega_{m}=\left[x_{1}, x_{m-1}\right] \times\left[y_{1}, y_{m-1}\right], \tilde{u}_{m}=f$ on $\partial \Omega$ and $\left|\nabla \tilde{u}_{m}-\nabla u_{m}^{*}\right| \leqslant C_{4} / m$, where constant $C_{4}$ is independent of $m$. Then

$$
\begin{aligned}
0 & \leqslant S\left(f^{L}\right)-S\left(u_{m}^{*}\right)=S\left(f^{L}\right)-S(f)+S(f)-S\left(\tilde{u}_{m}\right)+S\left(\tilde{u}_{m}\right)-S\left(u_{m}^{*}\right) \\
& \leqslant S\left(f^{L}\right)-S(f)+S\left(\tilde{u}_{m}\right)-S\left(u_{m}^{*}\right) \leqslant \frac{C_{1}}{m^{2}}+\iint_{\Omega \backslash \Omega_{m}}\left(\sqrt{1+\left|\nabla \tilde{u}_{m}\right|^{2}}-\sqrt{1+\left|\nabla u_{m}^{*}\right|^{2}}\right) d x d y \\
& \leqslant \frac{C_{1}}{m^{2}}+\frac{C_{4}}{m}(b-a)(d-c)\left(1-(1-2 / m)^{2}\right)=\frac{C_{1}}{m^{2}}+\frac{C_{5}}{m}\left(1-(1-2 / m)^{2}\right),
\end{aligned}
$$

where $C_{5}=C_{4}(b-a)(d-c)$. Therefore, it follows from Theorem 4 that

$$
\left|f-u_{m}^{*}\right| \leqslant\left|f-f^{L}\right|+\left|f^{L}-u_{m}^{*}\right| \leqslant\left|f-f^{L}\right|+4 P^{4 / 3}\left(\frac{S\left(f^{L}\right)-S\left(u_{m}^{*}\right)}{\lambda(\Omega) \pi}\right)^{\frac{1}{4}}
$$

where $P=\max \left\{1, P_{0}, P_{1}\right\}$. Applying the previous inequality, we obtain

$$
\begin{aligned}
\sup _{\Omega}\left|f(x, y)-u_{m}^{*}(x, y)\right| & \leqslant P_{0} \sqrt{(b-a)^{2}+(d-c)^{2}} \frac{1}{m}+4 P^{4 / 3}\left(\frac{\frac{C_{1}}{m^{2}}+\frac{C_{5}}{m}\left(1-(1-2 / m)^{2}\right)}{\lambda(\Omega) \pi}\right)^{\frac{1}{4}} \\
& \leqslant \frac{1}{m} P_{0} \sqrt{(b-a)^{2}+(d-c)^{2}}+4 P^{4 / 3}\left(\frac{\left.C_{1}+4 C_{5}\right)}{m^{2} \lambda(\Omega) \pi}\right)^{\frac{1}{4}} \rightarrow 0
\end{aligned}
$$

as $m \rightarrow \infty$.

\section{BIBLIOGRAPHY}

1. V.E. Mikhailenko, S.N. Kovalev. Construction of forms of modern architectural structures. Budivelnik, Kiev (1978). (in Russian).

2. A.A. Abdyushev, I.Kh. Miftakhutdinov, P.P. Osipov. Designing of nonslopping envelopes of minimal surface // Izvestia KazGASU. Building constructions, building and structures. 12:2, 86-92 (2009). (in Russian).

3. V.M. Mikljukov. On a new approach to Bernstein's theorem and related questions for equations of minimal surface type // Matem. Sbornik. 108(150):2, 268-289 (1979). [Math. USSR-Sbornik 36:2, 251-271.]

4. Jenn-Fang Hwang. A uniqueness theorem for the minimal surface equation // Pacific J. Math. 176:2, 357-364 (1996).

5. Jenn-Fang Hwang. How many theorems can be derived from a vector function - on uniqueness theorems for the minimal surface equation // Taiwanese J. Math. 7:4, 513-539 (2003).

6. R. Finn. Remarks relevant to minimal surfaces and to surfaces of constant mean curvature // J. d'Analyse Math. 14:1, 139-160 (1965).

7. T. Rado. The problem of the least area and the problem of Plateau // Mathem. Zeit. 32:1, 763-796 (1930).

8. S.N. Bernshtein. On equations of calculus of variations // Uspekhi Matem. Nauk. 8, 32-74 (1941). (in Russian). 
9. S.N. Bernshtein, I.G. Petrovskii. On the first boundary value problem (Dirichlet's problem) for equations of the elliptic type and on the properties of functions satisfying such equations // Uspekhi Matem. Nauk. 8, 8-31 (1941). (in Russian).

10. J. Serrin. The problem of Dirichlet for quasilinear elliptic differential equations with many independent variables // Phil. Trans. Royal Soc. London. A. 264:1153, 413-496 (1964).

11. G. Stampacchia. On some multiple integral problems in the calculus of variations // Comm. Pure Appl. Math. 16:4, 382-422 (1963).

12. H. Jenkins, J. Serrin. The Dirichlet problem for the minimal surface equation in higher dimension // J. Reine Angew. Math. 229, 170-187 (1968).

13. R.C. Bassanezi, U. Massari. The Dirichlet problem for the minimal surface equation in non-regular domains // Ann. Univ. Ferrara. 24:1, 181-189 (1978).

14. I.S. Berezin, N.P. Zhidkov. Calculation methods. V. 1. Gos. izd. fiz.-mat. liter., Moscow (1962). (in Russian).

15. A.A. Klyachin. On convergence rate of sequence minimizing area functional // Notes of Seminar "Superslow processes". 2, 136-142 (2007). (in Russian).

16. A.A. Klyachin. On convergence rate of sequence providing minimum in variational problem // Bull. Volgograd State Univ. Ser. 1. Math. Phys. 16:1, 12-20 (2012). (in Russian).

17. D. Gilbarg, N.S. Trudinger. Elliptic partial differential equations of second order. Springer, Berlin (1983).

Mikhail Andreevich Gatsunaev,

Volgograd State University,

Universitetsky av., 100,

400062, Volgograd, Russia

E-mail: mihpost@mail.ru

Alexei Alexandrovcih Klyachin, Volgograd State University,

Universitetsky av., 100,

400062, Volgograd, Russia

E-mail: klyachin-aa@yandex.ru 